\title{
A Robust Content-based Watermarking Technique
}

\author{
Mohammad Awrangjeb and Guojun Lu \\ Gippsland School of Information Technology, Monash University, Churchill, Vic 3842, Australia \\ \{Mohammad.Awrang jeb, Guojun. Lu\}@infotech.monash.edu.au
}

\begin{abstract}
Geometric transformations change the image pixel positions where the watermark is embedded. As a result, a copyright verifier fails to detect the watermark even though the watermark is present in the transformed image. The contentbased watermarking schemes try to solve this problem by first dividing the image into many disjoint patches and then locating those patches with respect to the salient points of the image. Most of the existing content-based schemes are vulnerable to scaling and general affine transformations. They do not work well against high JPEG compression either. This paper presents a contentbased blind watermarking scheme using corners. The proposed scheme achieves robustness against affine transformations and signal processing attacks by using triangular patch normalization and spread spectrum watermarking technique respectively.
\end{abstract}

\section{INTRODUCTION}

In image copyright protection, some pixels in the original image are used either to calculate the signature [1] or to embed the watermark [2]. However, geometric transformations change the location of those pixels. Consequently, the copyright verifier fails to track the copyright information in the transformed (test) image. This problem is known as the synchronization problem in the literature [3].

There are different types of synchronization schemes which aim to solve this problem [3]-[5]. In this paper, we will focus on the content-based synchronization schemes since they are promising [4], [5] and more robust than schemes of other types [6]. Kutter et al. named them as the second generation schemes in an earlier work [7].

In the content-based synchronization schemes, the image is first divided into many disjoint patches and their locations are defined with respect to the salient points of the image. Such salient points are denoted as the reference-points to the image patches. The watermark is then embedded into each patch separately. The copyright verifier can automatically locate those patches in the test image by detecting their referencepoints. A successful verification in at least one of the patches asserts the copyright ownership.

Nevertheless, locating the image patches is not always enough to solve the synchronization problem. The reason is, their area may be deformed under geometric transformations. For example, a simple $70 \%$ scaling attack shrinks a predefined circular area around a feature point. An anisotropic scaling changes the circular area (disk) into a parabolic area. As a result, the areas of the watermarked patches in the original and transformed images differ considerably and the copyright verifier may still fail to track the copyright information in the transformed patches, even though they are correctly located.
In order to overcome this problem, both the size and shape of the patches should be recognized and/or restored correctly.

Depending on the use of the original image information, the content-based synchronization schemes can be divided into two groups: semi-blind schemes and blind schemes. The semi-blind schemes use some of the information about the original image to achieve robustness against geometric transformations. For example, the works in [4], [8] matched the original and transformed feature-points for the recovery of the geometric distortions. The blind schemes [5], [9] achieve robustness against geometric transformations without using any information about the original image. Though the semiblind schemes are more robust than their blind counterparts, their applications are limited as the availability of the original information may be impractical.

In this paper, we present a robust content-based blind watermarking scheme. In Section II, we review the existing works. In Section III, we present the proposed watermarking scheme. In Section IV, we present our performance study and finally, in Section V, we conclude the paper.

\section{Previous Work}

Since the relative positions of the extracted feature-points from an image are geometrically invariant [10], any contentbased scheme automatically obtains robustness against translation and cropping attacks by referring the image patches with respect to the reference-points. Rotation invariance was achieved by using moment-based image normalization [11], [12]; disk alignment with its dominant gradient orientation [9], [13]; rotation correction [6]; polar-mapped circular patches [14]-[16]; and triangular patch normalization [5].

Though the schemes in [11], [13] were robust to rotation, they were vulnerable against scaling as the radius of the initial disks was a fixed value [6]. In order to achieve robustness against scaling the schemes in [6], [12], [14], [15] defined the radius of the circular patch in terms of the smoothing-scale at which the feature-point (center of the circle) was detected in the scale-space. Nevertheless, since the non-uniform scaling and shearing attacks change the circular patches into elliptical ones, the above methods are not robust to aspect-ratio changes and general affine transformations [14].

Tsai et al. [9] and Tone and Hamada [16] obtained nonuniform scaling invariance by normalizing the detected elliptical affine-regions around each feature-point into circular regions where the watermark was embedded. Both of the above methods were computationally intensive because of the use of iterative affine-region detection techniques. 
Bas et al. [5] is a more promising scheme where disjoint triangles were formed among the Harris points. The watermark (a pseudo-random sequence) is first arranged into the form of a standard isosceles right-angled triangle and then transformed back into the form of each original disjoint triangle. A watermarked triangle is simply calculated by adding each triangle with the corresponding transformed watermark triangle. In the detection phase, the watermarked triangle is transformed into the form of the standard triangle before verification.

The triangular patch normalization in [5] is more promising than the elliptical patch normalization in [9], [16] for the following reasons. First, triangular patches are formed among the feature-points. By normalizing them into the standard triangular patch the affine transformation invariance, including the rotation invariance, is directly achieved. In contrast, the elliptical patch is detected around each feature-point and, therefore, the rotation invariance must be separately achieved [9], [16], which may not always be obtained precisely [14]. Second, affine-region detection around the feature-points in the scale-space is a computationally expensive task.

However, the scheme in [5] has the following problems [6], [16]. First, the Harris detector is very much sensitive to noise and the detected interest-points suffer from high localization error. Second, the number of detected points, specially in the textured region, is very high. A smaller set of feature-points is usually selected from them so that the disjoint triangles are large enough for repeated embedding of the same watermark. The geometric distortions and noising seriously impede the selection of the same patch set in the transformed image. As a result, the sets of disjoint triangles in the original and transformed images differ significantly resulting poor overall performance of the watermarking scheme.

In this paper, we propose a content-based blind watermarking scheme similar to Bas et al. [5]. But we use corners detected by one of our previously proposed detectors [17] and error correction code to make the scheme more robust. Since corners are more robust than the interest-points, they offer better opportunity to survive under different attacks. The proposed scheme is much faster than the schemes using iterative affineregion detectors [9], [16]. Our comprehensive robustness study shows it more robust than the existing schemes [5], [9].

\section{Proposed Watermarking Scheme}

We first detect corners using our previously proposed contour-based multi-scale detector [17]. Then the Delaunay triangulations (DT) [18] are formed among the corners. Each triangle is normalized to a standard isosceles right-angled triangle. The watermark is repeatedly embedded into each normalized triangle using the direct-sequence spread spectrum communications technique [19]. The normalized and watermarked patch is de-normalized to the original shape and put back into the image. During watermark detection, we follow the same corner detection and patch normalization steps. Then the watermark is detected in each normalized patch.

In addition to achieving robustness against different attacks, there are two other essential requirements for successful wa- termarking. First, the embedded watermark should be perceptually invisible. Second, the false alarm probability $\left(P_{f a}\right.$, the probability of detecting the watermark in an un-watermarked image) should ideally be zero. In general, robustness and perceptual invisibility of the watermark are two opposite goals - the more the distortion is the higher the robustness. We achieve robustness by using triangular patch normalization and spread-spectrum watermarking technique. We also obtain perceptual invisibility by using the characteristics of the human visual system (HVS) [2] and show by theoretical analysis that $P_{f a}$ of the proposed watermarking scheme is very low.

\section{A. Corner Extraction and Patch Normalization}

The multi-scale detector in [17] detects corners on the extracted edges from an image. In order to achieve robust triangulations against cropping attacks, we follow a preprocessing step to exclude the corners that are detected near the image boundary. If the corners are detected densely in some region, we select the stronger corners based on the assumption that their probability of survival is higher in the attacked images. This avoids forming very small triangles which are not suitable for watermark embedding. We apply DT approach to obtain a set of disjoint triangles that are formed among the corners. Each triangular patch is then normalized using the nearestneighbor interpolation technique. We discuss corner detection, preprocessing, and patch normalization steps below.

1) Corner Detection: The previous content-based watermarking schemes used interest-points. In contrast to corners which are image's spatial locations where edge segments make significant angles, interest-points include not only corners, but also other image locations that have large gradients in all directions at a predetermined scale [20]. In general, corners have the following advantages over the interest-points:

- Corners are visually distinguishable and more robust than the interest-points.

- In an image, the number of corners is much lower than the number of interest-points. The lower number of corners is helpful for robust selection of patches of suitable sizes.

- Corners can be ranked based on their strength or the number of corners can be controlled by changing the edge detection thresholds. In contrast, it is hard to rank the interest-points.

- Corner detection requires less time than interest-point detection in the scale-space.

Therefore, we use corner detector instead of an interestpoint detector in the proposed watermarking scheme. We explain the working principle of our previously proposed CPDA (chord-to-point distance accumulation) corner detector [17] below.

The CPDA detector first extracts planar curves from the edge image detected by the Canny edge detector. Each curve is then smoothed with a small width Gaussian kernel in order to remove quantization noise and trivial details. In order to make strong and weak corners more distinguishable, we first use three chords of different lengths to estimate three normalized discrete curvature values on each point of the smoothed curve. 
Then we multiply the normalized curvatures to obtain the curvature product (a single estimated curvature) at each point. The maxima of the absolute curvature products along the smoothed curve are then obtained as candidate corners. Finally, it follows a two-step refinement process that removes weak and false corners using thresholds.

Let $C$ be the set of detected corners. For each corner the CPDA detector makes various information available, e.g., the corner position and the estimated curvature (product) value at this corner. We use the corner positions as the vertices of the triangular patches and the curvature values to rank the corners.

2) Preprocessing: We follow some preprocessing steps on the detected corners in order to obtain robust triangulations and patches of suitable sizes.

For achieving robustness against cropping attacks, we exclude the corners that are detected very close to the boundary of the image. If a corner is detected within $D_{b}$ pixels of the image boundary we remove it from $C$, where

$$
D_{b}=(w+h) / \rho,
$$

where $w$ and $h$ are width and height of the image and $\rho$ is an experimentally selected parameter. If $\rho$ is small, the required number of patches may not be preserved. If $\rho$ is large, the triangular patches will be affected even in small cropping. We set $\rho=24$ according to [5]. Consequently, For an image of size $512 \times 512, D_{b}=42$.

In order to avoid forming very small triangles which are not suitable for watermark embedding, we consider a circular region around each corner. The diameter of this region depends on the image dimensions and the total number of detected corners $|C|$ in the image. It is calculated as [8]

$$
D_{c}=\sqrt{\frac{w \times h}{n \times \beta}},
$$

where $n$ and $\beta$ are empirically selected parameters which are used to control the total number of selected corners from $C$ [8]. We use $\beta=60$ according to [8]. We set $n=1.5$ if $|C|>30$ or $n=2.5$ if $30 \leq|C|>15$ or $n=3.5$ otherwise. For an image with 20 detected corners, $D_{c}=42$. Qi and Qi [8] also set one of three above values for $n$ based on the texture of the image.

If there are more than one corners in the circular neighborhood of a corner, we select the strongest one having the highest estimated curvature. If more than one corners have the same curvature value, we select the one whose corresponding curve is the longest. These rules are based on the assumption that the stronger corners possess the higher probability of survival in image modifications and long curves are more likely to be detected than short curves when the image is down-scaled.

3) Patch Normalization: After detection and selection of corners, we employ the DT technique [18] to decompose the image into a set of disjoint triangles. The watermark is embedded (Section III-B) or detected (Section III-C) at each triangle after normalizing it to a standard triangle.

The DT has the following important properties [5]:

- Locality: If a vertex disappears, only the triangles associated to this triangle are affected.

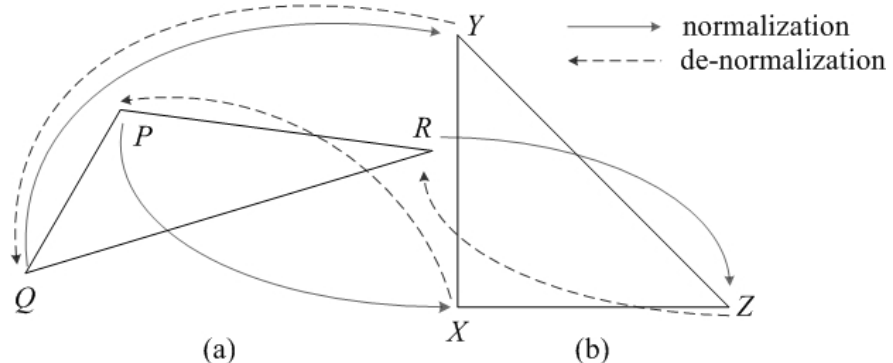

(a)

(b)

Fig. 1. Patch normalization and de-normalization processes: (a) a triangular patch in the image, (b) the standard patch $(64 \times 64$ isosceles right-angled $)$.

- Stability: Each vertex is stable within a specific area around it, when the triangulations is not affected even if the vertex moves within this area. Due to this property, the triangles are not affected in the presence of some localization error in the detected corners between original and transformed images.

- Low computational cost: DT algorithm can be applied using fast algorithms [18].

The triangular patches obtained using the DT technique are of different sizes. Geometric transformations may also change their sizes in the watermarked image. However, the size of the watermark is fixed. Therefore, in order to facilitate robust watermark embedding and detection, the triangular patch has to be normalized to a standard size. We adopt a $64 \times 64$ isosceles right-angled triangle as standard [5]. Fig. 1 shows normalization (solid arrows) and de-normalization (dotted arrows) processes. Normalization denotes transformation of a triangular patch $\triangle P Q R$ in the image into the standard patch $\triangle X Y Z$. De-normalization is the reverse process where $\triangle X Y Z$ is transformed back into $\triangle P Q R$.

There are 6 different affine transformations that map a triangle into another triangle. To find a unique transformation, we sort the angles of $\triangle P Q R$ into descending order. Let $P, Q$, and $R$ be the sorted angles. They are mapped into angles $X$, $Y$, and $Z$ respectively during normalization, as shown in Fig. 1 . The reverse mapping takes place during de-normalization.

\section{B. Watermark Embedding}

For an original image, we first pass the image through a low pass filer which provides additional robustness against signal processing attacks [5]. We then follow corner detection, preprocessing, and patch normalization steps discussed above. We embed an $M$-bit message repeatedly in each normalized triangular patch using the direct-sequence spread spectrum communications technique [19]. The watermarked patch is denormalized to the original shape and put back into the image.

We achieve robustness against affine transformations and signal processing attacks by using patch normalization and spread spectrum watermarking respectively. The perceptual invisibility is achieved by using the HVS model [2]. Fig. 2 shows the different steps of the watermark embedding phase. Let us denote

$$
a_{j}, \quad a_{j} \in\{-1,+1\}, 1 \leq j \leq M
$$




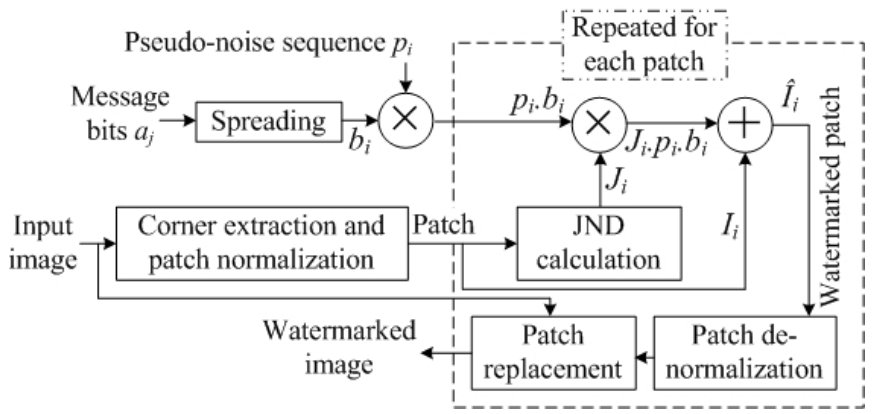

Fig. 2. Watermark embedding.

as a sequence of information bits we want to embed into a normalized triangular patch. In our case, the message $a_{j}$ is initially an $M=16$ bit binary number (owner's ID or a secret number) with 2-bit error correction capability using the $\mathrm{BCH}$ encoder [21] and we replace 0's with -1 's. We then spread this discrete signal $a_{j}$ by a large factor $c r$, called the chip-rate, and obtain the spread sequence

$$
b_{i}=a_{j}, \quad j . c r \leq i<(j+1) . c r .
$$

Fig. 3(a) shows an example of bit spreading. Spreading improves the robustness against attacks such as cropping, possibly due to patch overlapping. The number of pixels in the normalized patch is 2080 (all the pixels in the lower triangular patch shown in Fig. 3(b), including the diagonal pixels) and, therefore, we set $c r=130$. The spread sequence $b_{i}$ is modulated with a binary pseudo-noise sequence

$$
p_{i}, \quad p_{i} \in\{-1,+1\}
$$

and amplified with the JND (just noticeable distortion) sequence $J_{i}$ of the pixel sequence $I_{i}$. So the watermark

$$
w_{i}=J_{i} \cdot p_{i} \cdot b_{i} \text {. }
$$

To calculate the JND of the normalized triangular patch we follow the block-based HVS model used in [2]. The mirror of the normalized triangular patch is first produced as shown in Fig. 3(b). The original and mirror patches make a $64 \times 64$ square patch. The square patch is divided into $8 \times 8$ blocks and the block JND is calculated for each of these blocks by edge and texture analysis. Finally, the JND $J_{i}$ of each pixel is calculated using its luminance value $I_{i}$ and the corresponding block JND value. We need to calculate $J_{i}$ values only for the pixels in the lower triangular patch.

Finally, the watermarked pixel-sequence is obtained as

$$
\hat{I}_{i}=I_{i}+w_{i} .
$$

The watermarked patch is de-normalized and put back into the image. Consequently, we obtain the watermarked image.

\section{Watermark Detection}

For a given test image, we first follow corner detection, preprocessing, and patch normalization steps discussed in Section III-A. We then extract the embedded message bits from each normalized triangular patch. The extracted message

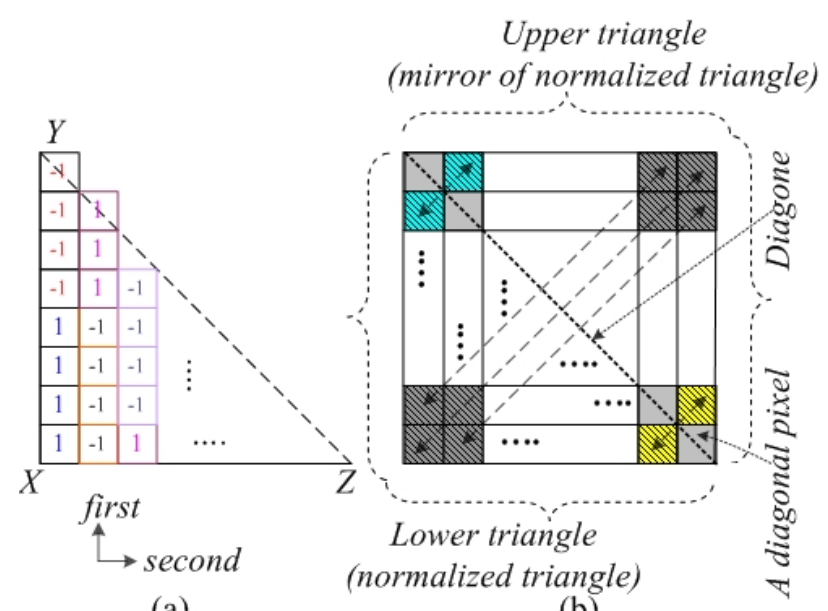

(a)

(b)

Fig. 3. (a) An example of bit spreading. A 5-bit message-sequence $\{1,-1,-1,1,-1\}$ is spreaded with a chip-rate 4 . The spreaded message is first repeated vertically ( $X$ to $Y$ ) and then horizontally ( $X$ to $Z$ ). (b) Producing mirror of the normalized triangular patch which is a $64 \times 64$ isosceles right-angled triangle. The original and mirror patches make a $64 \times 64$ square patch.

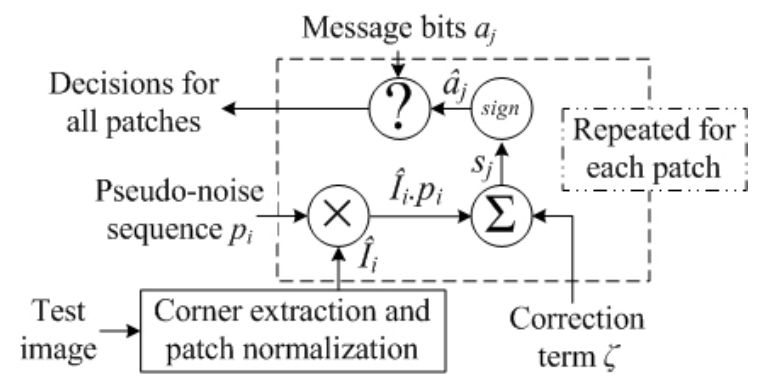

Fig. 4. Watermark detection.

is compared with the original message. If the difference is less than a threshold (as discussed later) for at least one patch in the test image, the ownership of the test image is declared in favor of the person who holds $a_{j}$ and $p_{i}$. Fig. 4 shows different steps of the detection phase. We detail them below.

Due to noisy nature of $p_{i}, w_{i}$ is also a noise-like signal and thus difficult to detect, locate, and manipulate. The recovery of the hidden information is easily accomplished by multiplying $\hat{I}_{i}$ with the same $p_{i}$ used in the embedding phase [19]:

$$
s_{j}=\sum p_{i} \cdot \hat{I}_{i}=\sum p_{i} \cdot I_{i}+\sum J_{i} \cdot p_{i}^{2} \cdot b_{i} .
$$

In the ideal case, when $p_{i}$ contains as many -1 's as 1 's in the summation interval $[j . c r \cdots(j+1) . c r-1], p_{i}$ and $I_{i}$ are uncorrelated and, therefore, the first summation term in the right-hand side of (8) equals to 0 . In practice, however, this is not the case and a correction term

$$
\zeta=-\operatorname{mean}\left(\hat{I}_{i}\right) \cdot \sum p_{i}
$$

is added to (8). So $s_{j}$ becomes

$$
s_{j}=\sum p_{i} \cdot \hat{I}_{i}+\zeta \approx c r . J_{i} . a_{j}
$$


TABLE I

FALSE ALARM PROBABILITy $P_{f a}$ WITH MINIMUM NUMBER OF MATCHING BITS $k$ AND MINIMUM NUMBER OF CORRECTLY DETECTED PATCHES $r$ THE TOTAL NUMBER OF EMBEDDED BIT IS $M=16(k \leq 16)$.

\begin{tabular}{c||c|c|c}
\hline \hline & $r=1$ & $r=2$ & $r=3$ \\
\hline \hline$k=14$ & $4.1 \times 10^{-2}$ & $8.1 \times 10^{-4}$ & $1.0 \times 10^{-5}$ \\
\hline$k=15$ & $5.2 \times 10^{-3}$ & $1.3 \times 10^{-5}$ & $2.0 \times 10^{-8}$ \\
\hline$k=16$ & $3.1 \times 10^{-4}$ & $4.4 \times 10^{-8}$ & $4.1 \times 10^{-12}$ \\
\hline \hline
\end{tabular}

and the recovered message bit $\hat{a}_{j}$ is

$$
\hat{a}_{j}=\operatorname{sign}\left(s_{j}\right) \text {. }
$$

After extracting $M$ message bits from a normalized patch, we follow an error correction step using the $\mathrm{BCH}$ decoder [21]. The corrected message is then compared with the original message. If at least $k$ number of bits $(k \leq M)$ match for at least $r$ number of patches in the test image, the ownership of the image is declared in favor of the person who holds $p_{i}$. We set $k=15$ and $r=1$ with a false alarm probability $P_{f a}=5.2 \times 10^{-3}$ (see Section III-D). Consequently, we allow an error rate $6.25 \%$ (1 out of 16), while Tsai et al. [9] allowed a $30 \%$ error. If necessary, a much lower $P_{f a}$ can be obtained by increasing $k, r$, or $M$, as we discuss in the next section.

Note that in the above watermarking technique even if someone knows the embedding and detection algorithm, he cannot extract the message as he does not know $p_{i}$ [19].

\section{False Alarm Estimation}

A false alarm happens when there is no watermark embedded in a test image, but the detector detects one. For a successful watermarking scheme, it is very important that its false alarm probability $P_{f a}$ should be as low as possible.

Let us assume a false alarm occurs if at least $k$ number of bits match for at least $r$ number of patches in the unwatermarked image, where an extracted bit can be considered as an independent random variable with the equal probability $(0.5)$ of being 0 and 1. Using Bernoulli's trials [22], the probability of successful watermark detection in a patch is

$$
P_{p}=\sum\left(\begin{array}{c}
M \\
u
\end{array}\right) \cdot(0.5)^{u} \cdot(0.5)^{M-u}, \quad k \leq u \leq M
$$

and, therefore, the probability of successful watermark detection $\left(P_{f a}\right)$ in an unwatermarked image is

$$
P_{f a}=\sum\left(\begin{array}{c}
N \\
v
\end{array}\right) \cdot\left(P_{p}\right)^{v} \cdot\left(1-P_{p}\right)^{N-v}, \quad r \leq v \leq N,
$$

where $N$ is the number of patches. For $N=20$, Table I shows theoretical $P_{f a}$ values for different $k$ and $r$. We observe that $P_{f a}$ decreases exponentially with slight changes in $k$ and $r$ values. Therefore, depending on the application, a suitable $P_{f a}$ can be chosen without changing $k$ and $r$ values much.

\section{EXPERIMENTAL RESULTS AND DISCUSSIONS}

We implemented the proposed watermarking scheme using MATLAB 7 and tested on many images. We compare the proposed scheme with Tsai et al. [9] and Bas et al. [5]. The

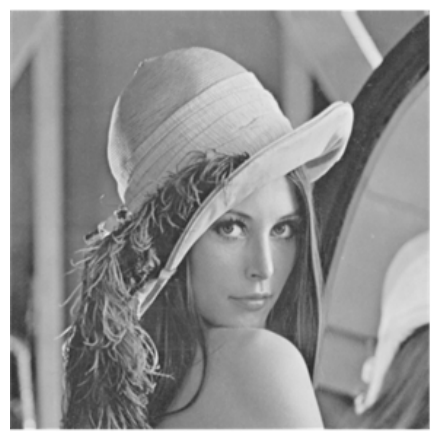

(a)

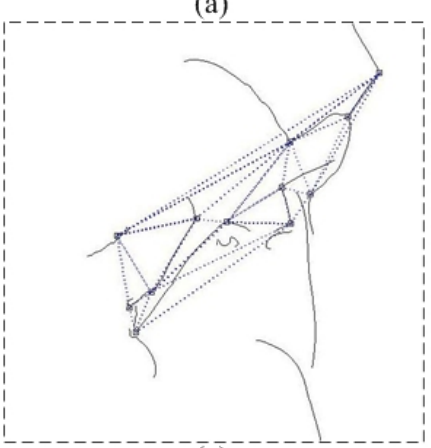

$(\bar{c})$

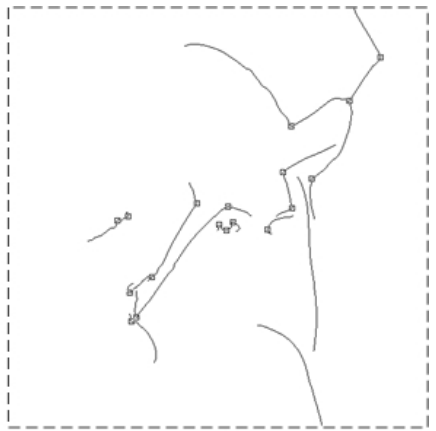

(b)

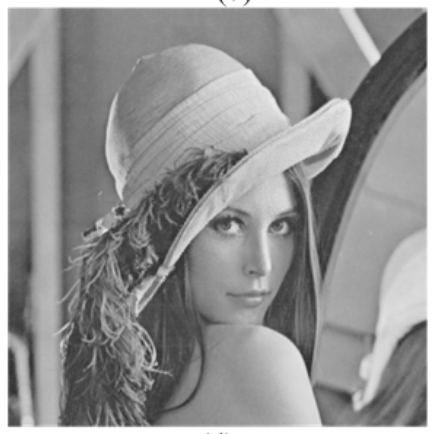

(d)
Fig. 5. Example of watermarking: (a) original 'Lena' image, (b) Extracted edges and detected corners using the CPDA detector [17], (c) Delaunay Triangulations formed among the selected corners. Corners that are shown in (b), but not in (c), were removed by preprocessing, (d) watermarked image.

reason of selecting these schemes [5], [9] are as follows. First, they are robust to affine transformations as discussed in Section II. Second, Tsai et al. presented detailed experimental results which we can use directly for comparisons. Third, Tsai et al. performed better than Seo and Yoo [15] and Tang and Hang [11] schemes. Since Tsai et al. presented detailed results on popular 'Lena' and 'Baboon' images, we present and compare results on these two images here. Most of the attacks were carried out using Stirmark benchmark [23].

Fig. 5 shows different phases of the proposed watermarking scheme using 'Lena' image. The PSNR (peak signal-to-noise ratio) between the original and watermarked images was $39.08 \mathrm{~dB}$. For 'Baboon' image the PSNR was $28.07 \mathrm{~dB}$. Since 'Baboon' is a highly textured image its JND values were higher and they caused higher changes in pixel values through watermarking. However, as we considered the HVS model [2] for calculating the JND values the watermarked images were perceptually similar to the original images.

Table II $^{1}$ shows the detection ratio which is the number of correctly detected patches in the attacked image divided by the number of watermarked patches in the original image. We observed that the proposed scheme was more robust to both geometric transformations and high JPEG compression (as low as a quality factor 10). It also offered better performance in nosing and filtering attacks than [5], [9].

\footnotetext{
${ }^{1}$ rotation attacks in our case were followed by a cropping so that the outer black parts were disappeared. In the case of [9], cropping did not occur. The better performance by [9] in high rotations was due to this reason.
} 
TABLE II

THE COMPARISONS OF DIFFERENT WATERMARKING SCHEMES USING DETECTION RATIO. THE SYMBOL '-' DENOTES UNAVAILABILITY OF DATA. PLEASE REFER TO [23] FOR DETAIL OF EACH ATTACK.

\begin{tabular}{|c|c|c|c|c|c|c|}
\hline \multirow{2}{*}{ Attacks $^{1}$} & \multicolumn{3}{|c|}{ 'Lena' image } & \multicolumn{3}{|c|}{ 'Baboon' image } \\
\hline & Ours & [9] & [5] & Ours & [9] & {$[5]$} \\
\hline Rot.-crop $1^{\circ}$ & $4 / 16$ & $5 / 19$ & - & $6 / 24$ & $6 / 25$ & - \\
\hline Rot.-crop $2^{\circ}$ & $4 / 16$ & - & - & $6 / 24$ & - & - \\
\hline Rot.-crop $5^{\circ}$ & $6 / 16$ & $5 / 19$ & - & $6 / 24$ & $4 / 25$ & - \\
\hline Rot.-crop $10^{\circ}$ & $4 / 16$ & - & - & $3 / 24$ & - & - \\
\hline Rot.-crop $20^{\circ}$ & $2 / 16$ & - & - & $1 / 24$ & - & - \\
\hline Rot.-crop $30^{\circ}$ & $4 / 16$ & - & - & $1 / 24$ & - & - \\
\hline Rot.-crop $45^{\circ}$ & $0 / 16$ & $2 / 19$ & $9 / 39$ & $2 / 24$ & $2 / 25$ & $2 / 40$ \\
\hline Rot.-scaling $1^{\circ}$ & $8 / 16$ & $3 / 19$ & - & $4 / 24$ & $9 / 24$ & - \\
\hline Rot.-scaling $2^{\circ}$ & $8 / 16$ & - & - & $3 / 24$ & - & - \\
\hline Rot.-scaling $5^{\circ}$ & $8 / 16$ & - & - & $5 / 24$ & - & - \\
\hline Rot.-scaling $10^{\circ}$ & $3 / 16$ & - & - & $1 / 24$ & - & - \\
\hline Rot.-scaling $20^{\circ}$ & $4 / 16$ & - & - & $0 / 24$ & - & - \\
\hline Cropping $90 \%$ & $7 / 16$ & $8 / 19$ & - & $8 / 24$ & $8 / 25$ & - \\
\hline Cropping $75 \%$ & $4 / 16$ & $5 / 19$ & $1 / 39$ & $3 / 24$ & $3 / 25$ & $0 / 40$ \\
\hline Cropping 50\% & $1 / 16$ & $2 / 19$ & - & $1 / 24$ & $2 / 25$ & - \\
\hline Affine 5 & $8 / 16$ & - & - & $2 / 24$ & - & - \\
\hline Affine 6 & $3 / 16$ & $9 / 19$ & - & $5 / 24$ & $14 / 25$ & - \\
\hline Affine 7 & $7 / 16$ & $5 / 19$ & - & $2 / 24$ & $12 / 25$ & - \\
\hline Affine 8 & $7 / 16$ & $8 / 19$ & - & $5 / 24$ & $10 / 25$ & - \\
\hline Aspect-ratio $0.8,1.0$ & $2 / 16$ & $1 / 19$ & $2 / 39$ & $3 / 24$ & $2 / 25$ & $0 / 40$ \\
\hline Aspect-ratio 1.0,1.1 & $2 / 16$ & $2 / 19$ & - & $2 / 24$ & $8 / 25$ & - \\
\hline Scaling $50 \%$ & $1 / 16$ & $1 / 19$ & $0 / 39$ & $2 / 24$ & $0 / 25$ & $0 / 40$ \\
\hline Scaling $75 \%$ & $4 / 16$ & - & $0 / 39$ & $3 / 24$ & - & $0 / 40$ \\
\hline Scaling 90\% & $7 / 16$ & $2 / 19$ & - & $0 / 24$ & $2 / 25$ & - \\
\hline Scaling $110 \%$ & $3 / 16$ & $4 / 19$ & - & $2 / 24$ & $4 / 25$ & - \\
\hline Scaling $150 \%$ & $6 / 16$ & - & - & $1 / 24$ & - & - \\
\hline Scaling $200 \%$ & $5 / 16$ & - & - & $1 / 24$ & - & - \\
\hline Rand.-dist. 0.95 & $10 / 16$ & $4 / 19$ & $16 / 39$ & $2 / 24$ & $0 / 25$ & $5 / 40$ \\
\hline Rand.-dist. 1.0 & $5 / 16$ & - & - & $4 / 24$ & - & - \\
\hline Rand.-dist. 1.05 & $4 / 16$ & - & - & $3 / 24$ & - & - \\
\hline Rand.-dist. 1.10 & $5 / 16$ & - & - & $2 / 24$ & - & - \\
\hline Row-col. rem. 5,5 & $7 / 16$ & - & - & $1 / 24$ & - & - \\
\hline Row-col. rem. 2,6 & $3 / 16$ & $6 / 19$ & - & $1 / 24$ & $9 / 25$ & - \\
\hline Row-col. rem. 4,16 & $5 / 16$ & $3 / 19$ & - & $1 / 24$ & $9 / 25$ & - \\
\hline JPEG 10 & $4 / 16$ & - & - & $3 / 24$ & - & - \\
\hline JPEG 15 & $3 / 16$ & - & - & $1 / 24$ & - & - \\
\hline JPEG 20 & $4 / 16$ & $0 / 19$ & - & $2 / 24$ & $1 / 25$ & - \\
\hline JPEG 30 & $5 / 16$ & - & $4 / 39$ & $3 / 24$ & - & $3 / 40$ \\
\hline Median Filer $3 \times 3$ & $3 / 16$ & $1 / 19$ & $13 / 39$ & $2 / 24$ & $3 / 25$ & $0 / 40$ \\
\hline Median Filer $5 \times 5$ & $4 / 16$ & - & - & $1 / 24$ & - & - \\
\hline Median Filer $7 \times 7$ & $2 / 16$ & - & - & $1 / 24$ & - & - \\
\hline Median Filer $9 \times 9$ & $0 / 16$ & - & - & $0 / 24$ & - & - \\
\hline Gaussian Filer $3 \times 3$ & $6 / 16$ & $3 / 19$ & $18 / 39$ & $2 / 24$ & $5 / 25$ & $0 / 40$ \\
\hline Gaussian noise 0.01 & $3 / 16$ & - & $1 / 39$ & $3 / 24$ & - & $5 / 40$ \\
\hline Gaussian noise 0.005 & $3 / 16$ & - & - & $1 / 24$ & - & - \\
\hline Gaussian noise 0.0005 & $4 / 16$ & - & - & $1 / 24$ & - & - \\
\hline Sharpening filter & $2 / 16$ & $2 / 19$ & $7 / 39$ & $4 / 24$ & $4 / 25$ & $0 / 40$ \\
\hline
\end{tabular}

\section{CONCLUSION}

We have proposed a robust content-based blind watermarking scheme. We extract corners using the CPDA detector [17]. We follow a preprocessing step to make the scheme more robust to cropping attacks. We obtain a set of disjoint triangles using the DT technique. Each triangular patch is then normalized. The message is embedded using the directsequence spread spectrum communications technique [19]. The watermarked patches are de-normalized and put back into their original positions to get the watermarked image.

By experiments we have shown that the performance of the proposed scheme is better than two promising schemes [5], [9]. Moreover, it was computationally much less expensive than [9] which used the expensive affine-region detector.

\section{REFERENCES}

[1] M. Awrangjeb and M. Murshed, "Robust signature-based geometric invariant copyright protection," in Proc. Int. Conf. on Image Processing, Atlanta, USA, Oct. 2006, pp. 1961-1964.

2] M. Awrangjeb and M. S. Kankanhalli, "Reversible watermarking using a perceptual model," SPIE Journal of Electronic Imaging, vol. 14, no. 1, pp. $013014.1-8$, Jan.-Mar. 2005.

[3] M. Awrangjeb, M. Murshed, and G. Lu, "Global geometric distortion correction in images," in Proc. Int. Workshop on Multimedia Signal Processing, Victoria, BC, Canada, Oct. 2006, pp. 435-440.

[4] J. S. Seo and C. D. Yoo, "Image watermarking based on invariant regions of scale-space representation," IEEE Trans. Signal Process., vol. 54, no. 4, pp. 1537-1549, Apr. 2006.

[5] P. Bas, J. M. Chassery, and B. Macq, "Geometrically invariant watermarking using feature points," IEEE Trans. Image Process., vol. 11, no. 9, pp. 1014-1028, Sep. 2002.

[6] X. Wang, J. Wu, and P. Niu, "A new digital image watermarking algorithm resilient to desynchronization attacks," IEEE Trans. Inf. Forensics Security, vol. 2, no. 4, pp. 655-663, Dec. 2007.

[7] M. Kutter, S. K. Bhattacharjee, and T. Ebrahimi, "Towards second generation watermarking schemes," in Proc. Int. Conf. on Image Processing, vol. 1, Kobe, Japan, Oct. 1999, pp. 320-323.

[8] X. Qi and J. Qi, "A robust content-based digital image watermarking scheme," Signal Processing, vol. 87, no. 6, pp. 1264-1280, Jun. 2007.

[9] J. S. Tsai, W. B. Huang, C. L. Chen, and Y. H. Kuo, "A featurebased digital image watermarking for copyright protection and content authentication," in Proc. Int. Conf. on Image Processing, vol. 5, San Antonio, Texas, USA, Sep. 2007, pp. 469-472.

[10] R. Hartley and A. Zisserman, Multiple view geometry in computer vision, 2nd ed. UK: Cambridge University Press, Mar. 2004

[11] C. W. Tang and H. M. Hang, "A feature-based robust digital image watermarking scheme," IEEE Trans. Signal Process., vol. 51, no. 4, pp. 950-959, Apr. 2003.

[12] X. Y. Wang, L. M. Hou, and J. Wu, "A feature-based robust digital image watermarking against geometric attacks," Image and Vision Computing, vol. xx, no. yy, available online from Nov. 2007.

[13] D. Zheng and J. Zhao, "A rotation invariant feature and image normalization based image watermarking algorithm," in Proc. Int. Conf. on Multimedia \& Expo., Beijing, China, Jul. 2007, pp. 2098-2101.

[14] H. Y. Lee, H. Kim, and H. K. Lee, "Robust image watermarking using local invaraint features," Optical Engineering, vol. 45, no. 3, pp. 037 002.1-11, Mar. 2006.

[15] J. S. Seo and C. D. Yoo, "Localized image watermarking based on feature points of scale-space representation," Pattern Recognition, vol. 37, no. 7, Jul. 2004.

[16] M. Tone and N. Hamada, "Affine invariant digital image watermarking using feature points," in Proc. RISP Int. Workshop on Nonlinear Circuit and Signal Processing, Hawaii, USA, Mar. 2005.

[17] M. Awrangjeb and G. Lu, "Robust image corner detection based on the chord-to-point distance accumulation technique," IEEE Trans. Multimedia, vol. xx, no. yy, p. accpeted, May 2008.

[18] M. de Berg, O. Cheong, M. van Kreveld, and M. Overmars, Com putational Geometry: Algorithms and Applications, 3rd ed. Berlin, Germany: Springer-Verlag, Mar. 2008.

[19] F. Hartung and B. Girod, "Digital watermarking of raw and compressed video," in Proc. Int. Symposium on Digital Compression Technologies \& Systems for Video Communications, Oct. 1996, pp. 205-213.

[20] D. G. Lowe, "Distinctive image features from scale-invariant keypoints," International Journal of Computer Vision, vol. 60, no. 2, pp. 91-110, Nov. 2004.

[21] S. B. Wicker, Error Control Systems for Digital Communication and Storage. NJ, USA: Prentice Hall, Feb. 1995.

[22] M. Evans, N. Hastings, and B. Peacock, Statistical Distributions, 3rd ed. New York, USA: Wiley, Jul. 2000

[23] F. A. P. Petitcolas, "Watermarking schemes evaluation," IEEE Signal Processing, vol. 17, no. 5, pp. 58-64, Sep. 2000. 